\title{
Entre el acercamiento y la desconfianza. La radio en la revista Caras y Caretas en la década de 1930
}

\author{
Between rapprochement and distrust. The radio in Caras y Caretas magazine in \\ the 1930s
}

\author{
Paula Martínez Almudevar \\ paulamalmudevar@gmail.com \\ Escuela Interdisciplinaria de Altos Estudios Sociales. \\ Universidad Nacional de San Martin / Universidad \\ Nacional Arturo Jauretche, Argentina
}

Recepción: 16 Noviembre 2020

Aprobación: 22 Marzo 2021

Publicación: 03 Mayo 2021

Cita sugerida: Martínez Almudevar, P. (2021). Entre el acercamiento y la desconfianza. La radio en la revista Caras y Caretas en la década de 1930. Anuario del Instituto de Historia Argentina, 21(1), e136. https://doi.org/10.24215/2314257Xe136

\begin{abstract}
Resumen: El presente trabajo indaga sobre los vínculos y las negociaciones que la sección radial del magazine Caras y Caretas configuró en torno a la radio durante la década de 1930, período marcado por el proceso de consolidación de la radiofonía en Argentina. Se propone abordar la sección Radio de dicha revistay reconstruir los múltiples diálogos y sentidos conformados sobre el desarrollo del fenómeno radial y las vinculaciones de la prensa gráfica con el nuevo medio. Podemos afirmar que la sección Radio contenía diálogos variados, en diversos planos y entre diferentes actores, en correspondencia con los múltiples intereses que reunían a lectores y redactores en una misma sección. Esto generó un pacto de lectura donde los intereses de las lectoras y los lectores debían ser tenidos en cuenta. El movimiento desde la crítica, la desconfianza y el supuesto "desinterés" hacia la firma de un contrato con la propia Radio Belgrano permite identificar la importancia que la radio tenía en la sociedad porteña de los años treinta y también cómo los intereses económicos y comerciales comenzaron a relacionar diferentes industrias culturales, como lo es la prensa escrita.
\end{abstract}

Palabras clave: Radiofonía, Prensa, Conexiones, Buenos Aires, Década de 1930.

Abstract: This paper investigates the links and negotiations that the radio section of the magazine 'Caras y Caretas' set up around radio during the 1930s, a period marked by the consolidation process of radio in Argentina. It intends to address the Radio section of said magazine, reconstructing the multiple dialogues and meanings configured on the development of the radio phenomenon and analysing the links between the graphic press and the new medium. We can affirm that the "Radio" section contained various dialogues, at different levels and between different actors, in correspondence with the multiple interests that brought together readers and editors in the same section. This formed a reading pact where the interests of the readers had to be taken into account. The movement from criticism, mistrust and the supposed "disinterest" towards the signing of a contract with Radio Belgrano itself allows us to identify the importance that radio had in Buenos Aires's society in the 1930s also showing how economic and commercial interests began to relate different cultural industries, such as the written press.

Keywords: Radio, Press, Connections, Buenos Aires, 1930s. 


\section{INTRODUCCIÓN}

La primera transmisión radiofónica tuvo lugar el 27 de agosto de 1920. Desde ese momento la radiofonía comenzó un largo camino que la transformaría, durante la década de 1930, en uno de los medios de comunicación y entretenimiento de masas más importantes del país. Ese recorrido y su posterior consolidación como industria cultural habilitó el desarrollo de empresas editoriales, como las revistas especializadas en la materia. Pero también impulsó la aparición de secciones en magazines populares que ya tenían un lugar relevante en la sociedad porteña de los años treinta y circulaban masivamente, como la revista Caras y Caretas.

La masividad que alcanzaba dicho magazine no se produjo por sí sola; para atraer a una gran variedad de lectores con intereses diferentes debió incorporar entre sus páginas las secciones y los temas que interesaban a un público amplio, y eso sucedió con la sección radiofónica. Esto trajo aparejado el surgimiento de una serie de diálogos, negociaciones y la construcción de un pacto de lectura específico entre los lectores y redactores, que variaba según la temática abordada.

Un producto cultural como Caras y Caretas, que fue popular y masivo durante la década del veinte, incorporó la sección radial a comienzos del siguiente decenio. A diferencia de su trayectoria cosmopolita previa, en este último desplegó y configuró ciertos argumentos cercanos a las ideas nacionalistas que comenzaron a circular durante la época, defendiendo valores culturales y nacionales por sobre los transmitidos por las emisoras. Es por ello que poner el foco en dicho objeto nos permitirá indagar en la dificultad de la revista, de, por un lado, discutir, dialogar y vincularse con las formas y contenidos que adquiere la radiofonía en ese momento, y, por otro, de responder a la curiosidad que había despertado en sus lectores.

Este texto examina el modo en que una empresa editorial como Caras y Caretas, ya consolidada en la década de 1930, comienza a vincularse y a establecer ciertos diálogos y negociaciones con esta novedad del período: la radio. A través del análisis de la sección "Radio" — que luego cambiará de nombre a "Radio Cocktail"—, incorporada a la revista a fines de 1933, se propone caracterizar este primer acercamiento del magazine al mundo radial, las representaciones y las críticas que suscita su funcionamiento, el contenido poco cultural y nacional de sus programas y las transformaciones de esas percepciones durante toda la década. Las discusiones sobre lo que la radio era y lo que debía ser, la calidad de sus programas, las valoraciones de las emisoras y las formas en las que debía transmitirse tenían lugar en la sección de una revista que no se especializaba en la temática radial.

Por estas características, la sección "Radio" se vuelve una fuente importante para preguntas sobre la historia cultural y social tales como ¿cuál es la posición que tiene Caras y Caretas sobre un nuevo medio como la radio?; ¿cuáles son las valoraciones que hace de su funcionamiento y de los programas emitidos?; ¿qué pacto de lectura se establece entre el medio y los lectores?; ¿qué tipo de intermediación cumple con sus "lectores radioescuchas"?

En un primer apartado se analiza la revista Caras y Caretas como un producto cultural ya consolidado dentro de la cultura de masas argentina, como también su posicionamiento explícito sobre la radiofonía. En el segundo apartado se caracteriza dicha sección, sus múltiples columnas y contenidos, así como las transformaciones y reestructuraciones internas producto de las diferentes percepciones que el magazine tenía del medio radial. Por último, se describen las transformaciones que la sección presenta a partir de 1936 en términos de su estructura interna y de la vinculación con el medio radiofónico. Este punto resulta interesante para entender la importancia que la radio comienza a tener en la sociedad porteña de los años treinta y también cómo los intereses económicos y comerciales empiezan a relacionar diferentes industrias culturales, como la prensa escrita. 


\section{Caras y Caretas y LOS "NUEvOS RITMOS DE LA ÉPOCA"}

Los habitantes de Buenos Aires en la década de 1930 consideraban que vivían en una ciudad moderna. Las transformaciones tecnológicas, las nuevas configuraciones urbanas y las novedosas formas que tomaban los medios masivos de comunicación eran la expresión de esos cambios. Este proceso de modernización no fue estable ni equilibrado, sino que evidenció las tensiones de la sociedad porteña de esos años (Caimari, 2012; Fara, 2020; Liernur, 2000).

La aparición y luego consolidación de la radiofonía puede considerarse como parte de ese proceso de transformación que no solo está relacionado con nuevas configuraciones del espacio urbano, sino también con nuevas formas de comunicación. En ese sentido, el surgimiento tanto de revistas especializadas en radio como de secciones dedicadas a ella dentro de las revistas de variedades que ya circulaban en la ciudad dan cuenta del interés que este medio despertaba en la sociedad porteña de los años treinta.

Una de estas últimas fue la revista Caras y Caretas, que desde comienzos de la década había empezado a incorporar algunas noticias y novedades sobre el medio, pero sin una sección específica. Con una extensa trayectoria, esta revista era una de las principales exponentes de la industria editorial del momento. En sus casi 35 años de vida había logrado adaptarse a los cambios de las épocas y su singularidad radicaba en la combinación de recursos gráficos y narrativos diversos, su capacidad tanto de informar como de entretener (Szir, 2009). Caras y Caretas fue el medio gráfico argentino de principios de siglo con mayor continuidad temporal (41 años), más cantidad de ediciones (2139 números) y mayor circulación. En todo ese período, las posturas del magazine respecto a diversos debates políticos y sociales fueron variando. Por ejemplo, mientras que a principios de siglo se percibía en la revista una relativa simpatía por los reclamos de los trabajadores, hacia 1919 Caras y Caretas tomó una postura claramente definida contra los obreros. En la cobertura de los hechos de la Semana Trágica, construyó un relato a favor de las fuerzas que reprimieron a los manifestantes, a los que llamó "minorías subversivas" (Yujnovsky, 2004, p. 137).

$\mathrm{Al}$ tratarse de una revista de circulación masiva, sus redactores debían interpelar a un público amplio y de variados - y distantes - intereses. Lo mismo sucedió con la nueva sección radial, incorporada a fines de 1933. Pero hay algo que diferencia al magazine de la década anterior y es el gran componente nacionalista en el espacio dedicado a la radio. La mirada sobre la calidad de los productos culturales, como también los valores nacionales de este período, van a ser determinantes en los diálogos y en la construcción del pacto de lectura que lleve adelante Caras y Caretas con un nuevo medio como la radio y sus lectores. La incorporación de dicha sección nos permitirá poner el foco en lo que representó la radiofonía y las tensiones que sus contenidos generaron internamente en el magazine.

En su primera aparición, la sección ocupó 27 páginas. Ya en el número anterior la revista anticipaba el sumario con las notas que figurarían en la misma:

\footnotetext{
Caricaturas en 5 colores por Valdivia, de LIBERTAD LAMARQUE, CARLOS GARDEL, MERCEDES SIMONE, TOMAS SIMARI e IGNACIO CORSINI

TEXTO:

"Nuestros propósitos". - "Los precursores de la radiotelefonía en el país, por Federico E. Bos, artículo ilustrado con caricatura, en 5 colores, del contraalmirante (R.) don Luis F. Orlandini, firmada por Valdivia. - "Lo que nos dicen. . Mercedes Carné, la Señorita Alegría, Juan de Dios Filiberto y León Fontova". - "Fadings", notitas cómicas. - "Las publicaciones argentinas para el radioescucha y las que interesan al radio- experimentador", nota gráfica. — "La radio y la evocación de la época rosista", por Julio Indarte, dibujos en 5 colores, dg Alvarez. - "Don Enrique Del Ponte, primer director artístico de broadcásting en la Argentina", recuerdos anecdóticos, por Contra de Tono, dibujo de Valdivia.

— " ? " . - "Los receptores modernos", dibujos en colores y características de los nuevos aparatos radiotelefónicos que funcionan en el país. - "La primera carta", colaboración anónima firmada con el seudónimo A. Vispa. — "Quince minutos
} 
con los actores Casares Pearson- Walk", por Car/os Ponce, caricatura en colores, de Valdivia. — "Interrupciones molestas". - "Noticias de las broadcástings".

SECCIÓN GRÁFICA, IMPRESA EN PAPEL GLASEADO

"Actuantes en el micrófono", retratos de los principales cantantes, comentaristas, recitadores, pianistas, violinistas, etc. "Conjuntos teatrales", grupos fotográficos de conocidos artistas. — "La Asociación de Broad- casters Argentinos", retratos de sus autoridades. - "Broadcasters conoci- dos". - "Autores radioteatrales". - "Directores artísticos de las radiodifusoras".- "Speakers". - "Orquestas y sus directores". Esta sección trae retratos de actuantes de la Capital, Rosario, Bahía Blanca y Montevideo (Caras y Caretas, 1933, N 1834).

Las notas de esta primera entrega se hallaban junto a caricaturas de los principales artistas del medio y ya para el final, la sección gráfica incluía las fotografías de todo aquel que participara del ambiente radiofónico. El primer artículo expuso sus propósitos:

"Caras y Caretas", consecuente con la invariable norma de reflejar en sus páginas todo cuanto signifique nuevos ritmos de la época, no reñidos con las buenas costumbres ni con la cultura del país, se complace en presentar a sus lectores radioescuchas esta nueva sección de radio... (Caras y Caretas, 1933, N 1834).

A primera vista, parecería que sus redactores estaban entusiasmados con reflejar los "nuevos ritmos" de la época, en tanto no atentaran contra "las buenas costumbres y la cultura del país". El temor a que los programas fueran una afrenta para el "buen gusto" o la moralidad de la época, o que no se ajustaran a la cultura nacional, suponiendo la incorporación de contenidos extranjeros, nos anticipa la valoración de Caras y Caretas respecto a la programación del nuevo medio.

Como en otros campos, la aparición de la radio y su rápida penetración en los hogares de la ciudad de Buenos Aires expusieron las tensiones que generaba el proceso de modernización en el país. Quienes se encargaban de la sección Radio de Caras y Caretas no eran ajenos a esta realidad, como se percibe en otro de los pasajes del ya citado primer artículo de la sección donde se puede leer: "Nos proponemos, pues, dedicar a la radiotelefonía toda la atención que se merece como medio de cultura, pero trataremos también, sin ahorrar esfuerzos, de encauzarla en rumbos decisivos hacia un nacionalismo amplio y puro..." (Caras y Caretas, 1933, $\mathrm{N}^{\circ}$ 1835. Subrayado de la autora).

El nivel cultural y los contenidos de carácter nacional fueron el centro de atención de quienes escribían en dicha sección. La repetitiva mención de las palabras cultura y nacionalismo da cuenta de los sentidos y las representaciones que sus redactores buscaban defender y transmitir. Querer encauzar a la radiofonía hacia el rumbo del nacionalismo suponía la existencia de otro camino desdeñable, que se buscaba evitar. Asimismo, la preocupación porque los contenidos fueran de carácter nacional puede enmarcarse en la influencia de los discursos nacionalistas de la época, a tono con el nuevo período político abierto con el golpe de Estado del 6 de septiembre de 1930. El movimiento nacionalista fue un fenómeno de orden político y cultural que se preocupaba por las nuevas tecnologías culturales. En ese sentido, muchos intelectuales nacionalistas trascendieron los límites del propio movimiento para instalar en la sociedad ciertas nociones en forma perdurable (Rubinzal, 2012). Es posible pensar, entonces, que algunas de ellas fueron adoptadas por los redactores de la revista, al menos en la sección Radio, las cuales también se enmarcaron en un debate más amplio sobre el objetivo que debía tener la radio, los valores que debía reproducir e incluso su organización comercial. Sus precursores, "los locos de la azotea", habían desarrollado esta nueva tecnología con el fin de volver accesibles muchos productos culturales de la época. Pero a medida que la radio se expandía y se masificaba, sus programas, según los contemporáneos, se ajustaban más a las necesidades comerciales de las emisoras. Una problemática recurrente derivada de ella fue el uso masivo de avisos comerciales que la radio emitía para financiarse. Son varias las voces que aparecen en la sección de radio de Caras y Caretas sobre este tema, como la columna titulada "La intercalación de avisos" de la edición del 28 de abril de 1934, que se quejaba: 
(...) el abuso en la intercalación de avisos ha llegado ya a un punto que no es posible silenciar (...) Es el nuestro el único país del mundo donde se toleran estos hechos atentatorios contra la función primordial de la radiofonía: la cultura del pueblo (Caras y Caretas, 1934, $\mathrm{N}^{\circ} 1856$ ).

El tono de denuncia de dicho artículo da cuenta de las tensiones entre los denominados "avisos" - en otras palabras, la publicidad comercial - y el fin que debía tener la radiofonía de acuerdo con la visión expresada en la sección sobre la transmisión de programas culturales. De todas formas, resulta llamativa la posición de la revista porque ella misma se valió de los anuncios publicitarios para subsistir en sus primeros años de circulación. A pesar de las críticas de la elite intelectual, que observaba una tendencia materialista en esa decisión, Caras y Caretas se consolidó como la primera empresa editorial. Pero en la década de 1930, la existencia de estos artículos muestra cierta transformación, porque buscaban defender valores e ideas que alguna vez fueron acusados de transgredir (Rogers, 2003; 2005).

La radio comenzaba a estar en boca de todos, y pasaba a ocupar el centro de un debate que se desarrollaba en torno a la polarización entre su énfasis comercial o cultural. Como si la historia se repitiera, la emergencia de un nuevo medio hacía resurgir viejos debates acerca de qué debía decir un nuevo medio y cuál era la mejor forma de hacerlo. Cómo lidiar con elementos foráneos, con la deformación del lenguaje, con la producción de elementos de bajo nivel cultural y con la cantidad de avisos: todas estas eran parte de las preocupaciones que podemos observar en una Caras y Caretas que había optado, a principios del siglo XX, por los mismos recursos que criticaba. Dicha preocupación también comenzaba a verse en la clase política del momento. El propio director de Correos y Telégrafos, Carlos Risso Domínguez, en una entrevista a fines de 1933, declaraba tener el interés de que "los contenidos de la radio sean nacionales y de alto nivel cultural" (Caras y Caretas, 1933. No 1838). Podemos afirmar entonces que las preocupaciones de la revista y de los sectores políticos dan cuenta de una visión nacionalista y elitista de lo cultural. El hecho de que los programas debían ajustarse a dicha visión deja en claro las posiciones sobre "otra cultura" que no debía ser reproducida en el éter.

Pero las revistas especializadas y las secciones dedicadas al nuevo medio también debían satisfacer la curiosidad de sus lectores y lectoras, tratando de explicar lo que sucedía detrás de los parlantes. Responder a las preguntas de los y las oyentes implicaba para los redactores de la sección "Radio" volverse interlocutores válidos y negociar entre su propia agenda con tintes nacionalistas y los requerimientos del público, construyendo así un pacto de lectura que lo volviera posible.

\section{LAS DISCUSIONES EN LA SECCIÓN "RADIO”}

La historiadora Cecilia Gil Mariño argumenta que en este periodo se produjo el desarrollo de un modelo de producción de convergencia de medios en el cual la radio, el cine, la industria discográfica y de espectáculos construyeron un círculo virtuoso del consumo. Las nuevas revistas especializadas impulsaron la promoción de dichas industrias culturales (Gil Mariño, 2015). A diferencia de estas, la sección radial de Caras y Caretas no estaba motorizada por la necesidad de promocionar alguna emisora o programa en particular, cuya publicidad le diera rédito económico. Su interés radicaba, en un primer momento, en responder a la curiosidad social generada por este nuevo medio producto del avance de la técnica. Pero ese objetivo, como veremos a continuación, debía convivir con una agenda de corte elitista y nacionalista y generó la necesidad de establecer un pacto de lectura entre redactores y lectores.

La intención de este apartado es analizar las transformaciones de la sección "Radio" de Caras y Caretas y las múltiples problemáticas que presenta sobre el mundo radial de la época, los programas, los empresarios radiales y las preocupaciones de quienes escribieron en sus páginas durante la década de 1930. La radio, como un producto de la modernidad y del progreso técnico, necesitaba ser explorada y explicada. El interés de las notas en mostrar el funcionamiento de las emisoras y a sus protagonistas se vuelve significativo para pensar en la necesidad que tenían los y las radioescuchas de saber cómo funcionaba este flamante medio, qué sucedía tras las bambalinas radiales y quiénes participaban de ese nuevo mundo. Al mismo tiempo, la incorporación 
de este tipo de información en el magazine nos sugiere que la propia empresa editorial buscaba responder a los requerimientos del público, que si no encontraba estos contenidos entre sus páginas, podrían optar por leer Radiolandia o Antena, otras revistas especializadas en la radio.

En sus primeras apariciones, la sección ocupaba varias páginas, casi todas a color, con caricaturas de las principales actrices, los actores, las cancionistas y los directores artísticos de las mismas. Se visitaban emisoras, se escribía sobre la dinámica invisible de trabajo y producción en cada una de ellas y al mismo tiempo se detallaba quiénes eran los sujetos que hacían que la misma funcionara.

Para ilustrar un poco mejor esta idea vamos a enfocarnos en unos algunos artículos que Caras y Caretas incluyó entre las primeras apariciones de la sección radial. Se trata de un conjunto de notas donde se narra la cotidianidad de una tarde o una noche en la radio, a las que denominan "Las broadcastings vistas por dentro".

En una de estas recorridas por las radios porteñas, titulada "Una noche en Radio Prieto" (imagen 1) el cronista registra el ritmo de trabajo en la emisora, revelando sus protagonistas.

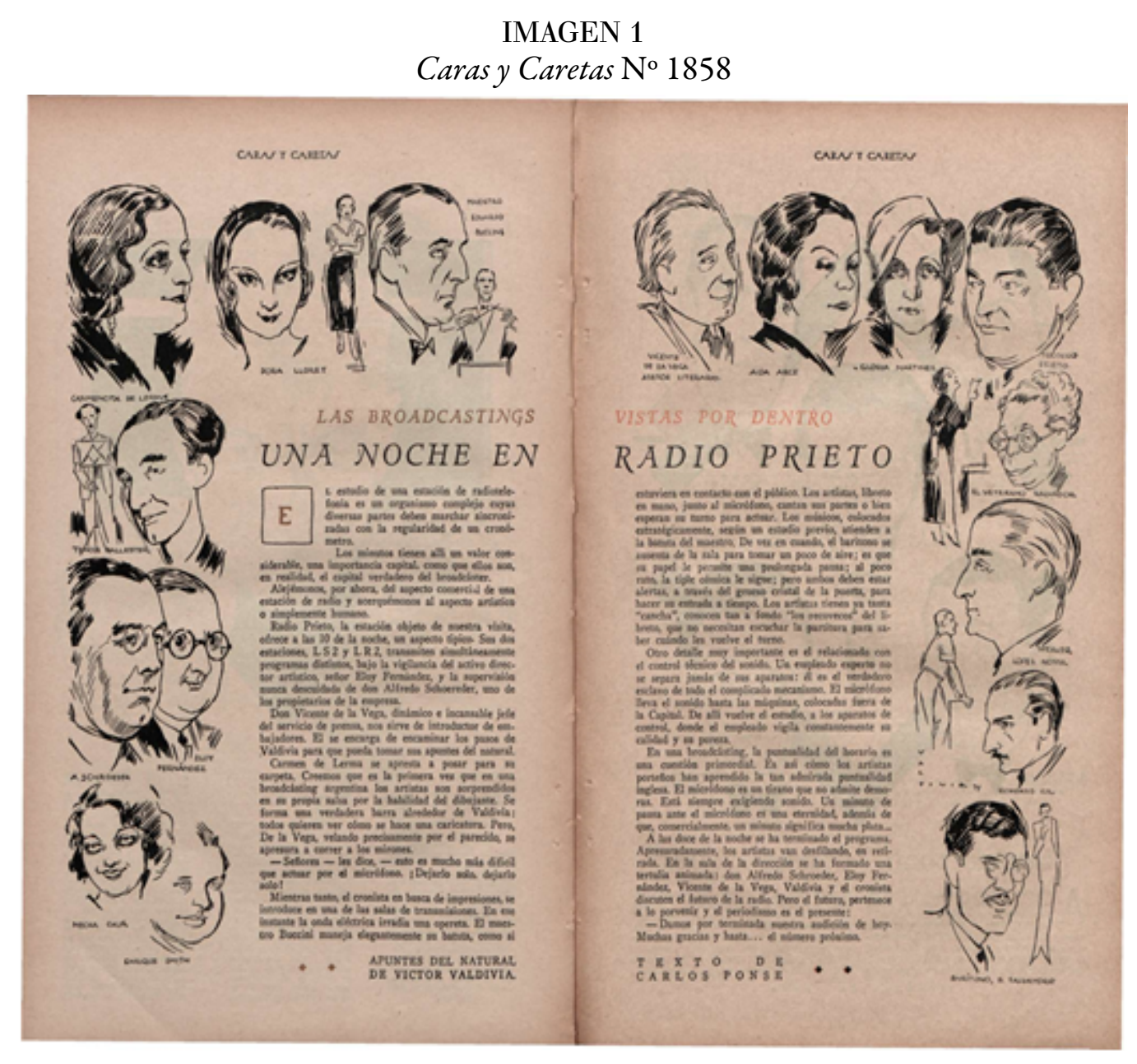

No faltan en estas narraciones la recepcionista, el director artístico, los dueños de la broadcasting (citados por la presencia del cronista en cuestión), los técnicos radiofónicos y, por supuesto, quienes hacen los programas, es decir, las y los artistas de radio y las orquestas. Cuando el cronista ingresa a la sala de transmisiones de Radio Prieto se encuentra con los y las artistas y las orquestas ya dispuestas, esperando para ejecutar su número porque:

En una Broadcasting la puntualidad del horario es una cuestión primordial. Es así como los artistas porteños han aprendido la tan admirada puntualidad inglesa. El micrófono es un tirano que no admite demoras. Está siempre exigiendo sonido. Un 
minuto de pausa ante el micrófono es una eternidad, además de que comercialmente un minuto significa mucha plata... (Caras y Caretas, 1934, № 1858).

La crónica presenta la complejidad del mundo radiofónico tanto en su organización técnica como también comercial. La imposición de un ritmo de trabajo propio de la radio expresaba las presiones económicas, debido a que había muchos números artísticos auspiciados por empresas que aprovechaban la temprana masividad de la radio para promocionar sus productos. Esos mismos avisos, a través de los cuales la radio se financiaba a sí misma, eran los que producían una gran preocupación en Caras y Caretas. Pero en esta crónica, la preocupación previa sobre los elementos exóticos y el "bajo nivel" cultural que resulta del énfasis comercial, se traduce en curiosidad y admiración por un desconocido, que expresa en palabras extranjeras como broadcasting, y la referencia a la puntualidad inglesa. La admiración e interés llevaban a una convivencia entre la postura desconfiada y crítica, y los elogios a ciertos programas. Por ello podía leerse en la misma página una columna como la siguiente: "No todo ha de ser de mala calidad en nuestra zarandeada radio. Hay cosas buenas, de bueno para arriba y nos place hacerlo constar asi”" (Caras y Caretas, 1934, N 1870).

Al final de la sección "Radio" eran habituales dos páginas denominadas "Radio Cocktail", donde se encontraban las valoraciones a los programas, las columnas más críticas y las novedades del medio. Algunas de ellas, como la columna “¡No me diga, che!”, marcaban un tiempo de lectura irónico, burlón y chismoso respecto a algunos eventos que sucedían en el mundo radial. En 1935, por ejemplo, se publicaba este comentario:

- Que todas las broadcastings han hecho fuego, como a una voz de mando, para comenzar la famosa "temporada".

- Que algunos "proyectiles" dieron en el blanco, pero que otros se perdieron en el vacío, es decir, hicieron "papa".

- Que muchos radioescuchas desean saber el porqué de la ausencia de Libertad Lamarque, la reina del tango.

- Que dicha ausencia se debe a un lío con cierto empresario, y que Libertad, amenazada por un formidable embargo, se ve obligada a no actuar en la República.

— Que el verdadero perjudicado con este lío teatro-judicial, es el público.

- Que estamos en pleno furor de estrenos, más o menos legítimos y más o menos verdaderos.

- Que la inauguración de la "temporada" ha venido a dar una inyección de vida a la desfalleciente radiotelefonía (Caras

$y$ Caretas, $\left.1934, \mathrm{~N}^{\circ} 1844\right)$.

El tono chismoso con el que Caras y Caretas menciona el comienzo de la temporada y la representación que hace de los programas radiales como si fueran "proyectiles" lanzados al público, contrasta fuertemente con las primeras páginas de la sección dedicadas a elogiar e ilustrar el star system radial. Cumpliendo con el pacto de lectura, se presenta además como defensora de los intereses del público, característica evidente en las aclaraciones a las inquietudes sobre la ausencia en el éter de Libertad Lamarque, reconocida actriz de teatro y cine que también se destacaba por cantar sus tangos en diferentes emisoras.

La observación, al final de la cita sobre la verdad de los estrenos sugiere el reaprovechamiento de programas y le da sentido al artículo que se encontraba a continuación, en la misma página, titulado "La desesperante uniformidad de los programas", donde se comentaba la similitud de los programas que se transmitían en las radios porteñas, en los cuales coincidían temáticas y horarios en diferentes puntos del dial: "Según puede verse, a ciertas horas no hay modo de librarse de ciertos números si no es silenciando el altoparlante, como sin duda lo harán miles de radioescuchas de buen gusto" (Caras y Caretas, 1934, N 1844).

La columna sobre la uniformidad de los programas finaliza con la frase "como sin duda lo harán miles de radioescuchas de buen gusto" (subrayado de la autora). Caras y Caretas construye ciertos sentidos respecto a la valoración de los programas de radio, estableciendo una jerarquía estética. Y llama a sus radioescuchas lectores a actuar de acuerdo con su propio criterio y gusto, rechazando una posición de consumo pasivo. Para comprender mejor el funcionamiento de las jerarquías y valoraciones, el recuadro titulado "El nivel semanal de las Broadcastings" (imagen 2) que señala la calidad de cada emisora que se encuentra en la ciudad, es revelador. Entre los niveles valorativos - de muy malo a muy bueno-, Caras y Caretas establece un 
ranking de quienes tenían los mejores programas, lo que solía coincidir con los programas recomendados y los “porotos" ganados, otro de los temas que abarcaba la sección radiofónica.

IMAGEN 2

Caras y Caretas, 7/3/36, № 1953

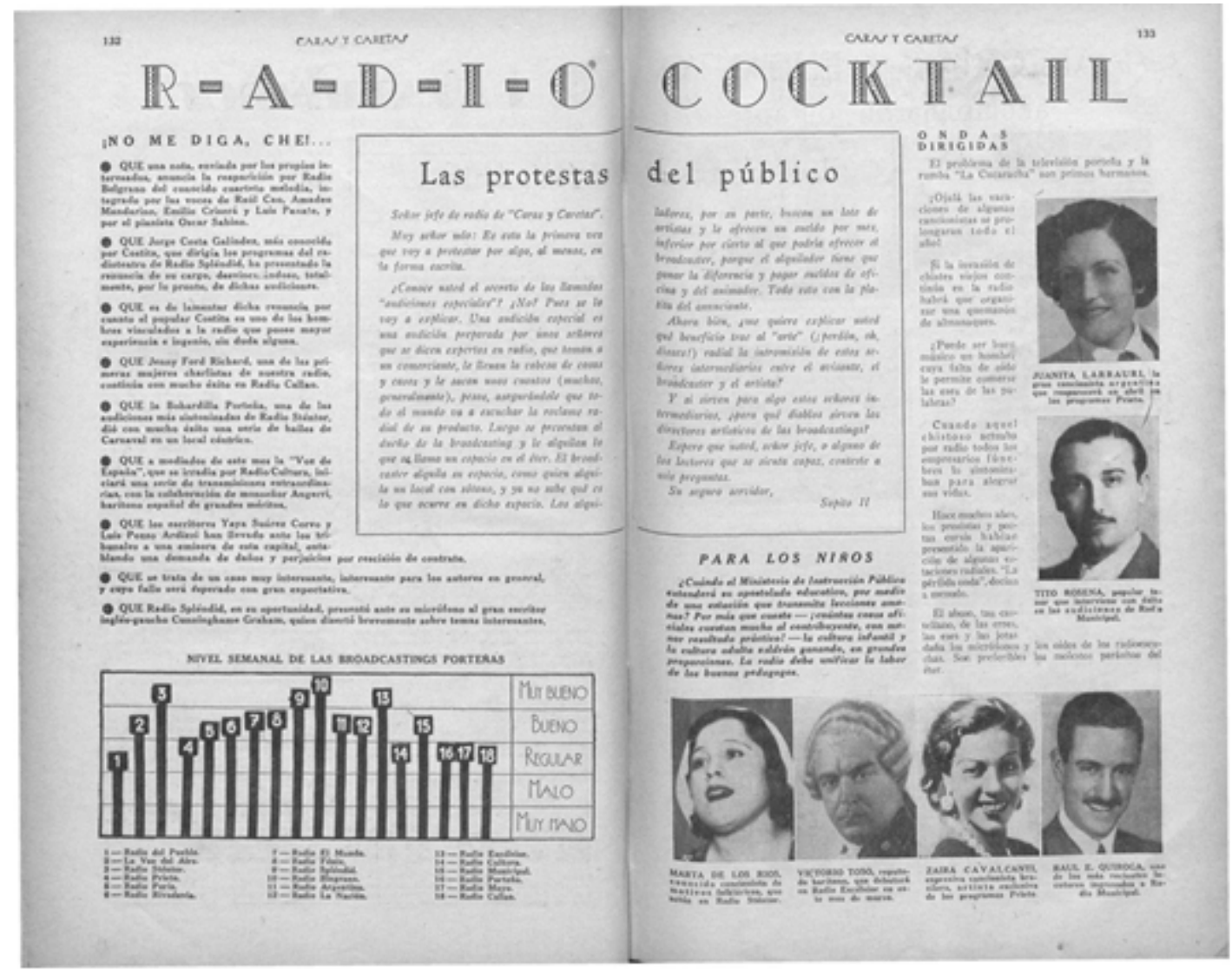

La apelación al "buen gusto" de los oyentes y la construcción de valoraciones a través de estos rankings son expresiones del pacto de lectura construido por el magazine con sus lectores, quienes debían posicionarse y actuar, como veremos a continuación - frente a los contenidos emitidos a través del éter.

En suma, aunque la radio se volvió un medio de comunicación de masas de gran popularidad, también generó preocupaciones y críticas, que pueden leerse en la prensa del momento. La sección Radio buscó comunicar y correr el velo de lo que sucedía tras bambalinas en las emisoras, pero en su interior, las discusiones estaban inmersas en la reafirmación de jerarquías y distanciamientos respecto a qué tipo de contenidos se debían divulgar y qué debían hacer las y los lectores frente a ello.

\section{Voces disonantes Y UNA NUEVA CONFIGURACión: "RAdio CockTAIL"}

Con el correr de los números la sección sufrió algunas transformaciones. Las primeras páginas que informaban sobre las novedades del star system radial con caricaturas a color y algunas fotografías desaparecieron, y solo quedaron las dos páginas más críticas de la sección que eran en blanco y negro y se titulaban "Radio Cocktail". Esta decisión editorial puede tener su explicación en la tensión que generaba contemplar la curiosidad y admiración de los lectores radioescuchas frente a un medio que se contraponía a la agenda y los valores estéticos elitistas expresados en Caras y Caretas. 
La avanzada de la radio sobre los hogares permitió llegar a muchas personas con un contenido que se volvía más masivo y estandarizado. Esto era evidente en la similitud de programas emitidos en las diferentes radios porteñas. Casi todas las emisoras de la ciudad habían optado por utilizar un formato con el cual se aseguraban una audiencia, al tiempo que generaban gran preocupación en la prensa especializada y, a veces, hasta en los propios oyentes. ${ }^{1}$

Los diferentes discursos sobre un mismo problema presentes en la sección responden a una característica propia de la prensa ilustrada de la época, por lo que podemos identificar que la revista Caras y Caretas presentaba en su sección Radio voces disonantes en la manera de informar y caracterizar la radio. ${ }^{2}$

Como dijimos, el fenómeno radiofónico debía ser explorado, desmenuzado y explicado a una gran cantidad de lectores del magazine interesados en él. Esas explicaciones no solo generaron discusiones y preocupaciones en cuanto a su expansión, también configuraron e hicieron visible - como lo demostró la crónica de la visita nocturna a Radio Prieto- un mundo social y laboral heterogéneo, al que se hicieron extensivas las discusiones sobre la forma que tomaba el medio.

Un ejemplo de ello es la aparición, en febrero de 1934, de este pequeño y revelador aviso:

Nada hay tan elocuente e irrefutable como la estadística. Es la ciencia exacta que jamás falla y que constituye la pesadilla de todos aquellos que no quieren o no les conviene encararse con la realidad. La sección Radio de CARAS Y CARETAS prepara para más adelante (por ejemplo, para la temporada oficial) una estadística con el cuadro exacto de los sueldos de los artistas de radio, con indicación de la broadcasting en que actúa, la cantidad de horas y otros detalles interesantes. La estadística se completará con la proporción cabal de aquellos artistas que reciben retribución y de aquellos que no la reciben. Los lectores podrán así hacerse una idea de la radio como arte y como medio de vida (Caras y Caretas, 1934, N $^{\circ} 1845$ ).

La revista enunciaba así, por única vez, la preocupación por dar a conocer a sus lectores las condiciones salariales de los y las artistas de radio. Y no solo eso: ponía en cuestión las representaciones del star system radial, dando cuenta de las diversas condiciones en las que el elenco artístico realizaba sus tareas en las emisoras.

Aunque la revista lo presentó como un dato muy promisorio, las estadísticas nunca aparecieron en la sección. Ello nos permite conjeturar sobre los problemas que su publicación podría haber causado en el medio. De todas formas, la promesa de su aparición no deja de ser un dato interesante que nos presenta una fotografía de la situación de los y las artistas de radio y de las preocupaciones que la revista encarnaba. Asimismo, la sección también informaba sobre los y las artistas contratados/as y hacía valoraciones respecto de los cambios a los que estaban sujetas esas contrataciones, como sucedía en la sección “iNo me diga, che!” que mencionaba lo siguiente:

-Que ahora parece que las broadcastings han abandonado, no sabemos si con carácter definitivo, a los NÚMEROS EXCLUSIVOS, dándose el caso de que una artista actúe todos los días de la semana, en una estación distinta.

-Que esta situación favorece francamente a los actuantes, siempre que la desaparición del "carácter exclusivo" no traiga una disminución de sus honorarios, en cuyo caso el artista tendría más trabajo pero menos retribución (Caras y Caretas, 1934, $\left.\mathrm{N}^{\circ} 1883\right)$.

Estas menciones a los cambios en las condiciones de trabajo de los y las artistas de radio exponen la atención que recaía sobre las consecuencias que estas modificaciones traían aparejadas a los sujetos en cuestión; quizás incluso expresaban una intención de quien escribía respecto a defender el trabajo artístico frente a estos cambios.

Por lo tanto, ¿dónde quedaron las preocupaciones por los contenidos de los programas radiales y la importancia de lo cultural y lo nacional?; ¿qué objetivo perseguían con las caracterizaciones del mundo del trabajo en la radio? La sección "Radio" no podía hacerse eco solamente de las cuestiones que versaban sobre los contenidos de las programaciones radiales, también debía cumplir con informar a sus lectores acerca de las novedades del mundo radial, lo que incluía la situación del mundo artístico que lo frecuentaba. 
La radiofonía era un fenómeno nuevo que debía ser traducido al público, y por esa razón Carasy Caretas no podía reducir su pacto de lectura con los lectores y lectoras a una mera crítica del funcionamiento del medio. La curiosidad que despertaban aquellos que participaban en él, cómo eran y qué hacían las y los principales artistas o las nuevas contrataciones de cada emisora eran cuestiones que también debían ser atendidas. Es por ello que podemos afirmar que dentro de la sección "Radio" se producían discusiones y diálogos múltiples, en diversos planos y entre diferentes actores, en correspondencia con los muchos y diferentes intereses que reunían a lectores y redactores en una misma sección. Pero, como mencionamos, el pacto de lectura construido por el magazine con sus lectores implicaba no solo informar acerca del star system, sino también posicionarse frente a los contenidos emitidos a través del éter.

Tal vez los momentos de mayor discusión e interacción entre relatores y público lector se producían cuando las grandes emisoras porteñas, antes de los inicios de las temporadas, se disputaban las mejores estrellas, con las cuales creían tener la garantía de audiencia asegurada. Es a partir de los artículos publicados en ese período que se hace explícito el pacto entre ambos. En 1935, por ejemplo, Caras y Caretas denunciaba que Radio Belgrano quería "quedarse con todas las estrellas" (Caras y Caretas, 1935, N 1935), a lo que titulaba "Trust de estrellas". Pero la contratación de estos astros no garantizaba la aceptación total por parte de la audiencia de los programas radiales, porque la crítica hacia la uniformidad de los mismos y a los y las artistas con menos experiencia excedía la propuesta artística de la emisora. Los críticos expertos lo hacían a través de artículos o pequeñas columnas y el público tenía su propio espacio: "Las protestas del público". En este último encontramos las quejas más duras contra aquella cancionista que solo aturdía o cuya voz no gustaba a los oyentes. En una de las cartas enviadas que aparecían en el espacio de "Las protestas del público", el firmante declaraba hablar "en nombre de cincuenta mil oyentes" (Caras y Caretas, 1935, $\mathrm{N}^{\circ}$ 1934) para criticar un tipo de género propiamente radiofónico que era el de las cancionistas, reflejado en lo que cantaba "Carmencita Silva”. En dicho género solían destacarse principalmente mujeres jóvenes que veían en la radio una oportunidad para comenzar su carrera como artistas. En cuanto género que abundaba en la radio, era considerado como el “... más implacablemente atacado por miles de aficionados. Destacarse es cosa difícil" (Caras y Caretas, 1933, $\mathrm{N}^{\circ}$ 1835). Eso mismo le sucedía al oyente que criticaba a Carmencita Silva, de quien decía “... a quien no tengo el honor de conocer ni por retrato" (Caras y Caretas, 1935, № 1934), dando cuenta así de la importancia de participar en los mecanismos de producción y difusión de imagen por los medios impresos en la construcción de las carreras artísticas.

La carrera abierta al talento, que tanto caracterizaba a la radio en Argentina y en otros países como EE. UU. y Uruguay (Halper, 2001; Ehrick, 2015), fue otro de los disparadores para que la prensa especializada y los radioescuchas dedicaran numerosos artículos y cartas a reclamar por el bajo nivel de los programas, cumpliendo nuevamente el rol de críticos y jueces implacables. La poca experiencia de algunos protagonistas de ciclos musicales o de radioteatros perjudicaba por completo a la programación. A veces, hasta los speakers recibían críticas por los errores en la modulación o conjugación de verbos. A pesar de que los empresarios de las broadcastings contrataban artistas reconocidos para actuar o cantar frente a sus micrófonos, las malas actuaciones, los diálogos insulsos y los tonos desafinados de quienes recién comenzaban con su carrera artística desanimaban a cualquier oyente. Por ejemplo, pese a que en la edición del 5 de octubre de 1935 se informó sobre el refuerzo del elenco de artistas de renombre en varias emisoras (Caras y Caretas, 1935, $\mathrm{N}^{\circ}$ 1931), los problemas persistían, ya que a principios de febrero de 1936, uno de los artículos expresaba:

¡Qué bueno sería!... Si los radioescuchas pudieran, como en el teatro, aplaudir o silbar a los que actúan ante el micrófono/ Si hubiera una comisión de censura para ciertas transmisiones donde se violan los más elementales principios del buen gusto (Caras y Caretas, 1936, N 1948).

Debajo de estas declaraciones aparecía una caricatura (imagen 3), recuperada de la conocida revista francesa Le Rire, que da cuenta de la valoración negativa que se tenía de los programas de radio. Aquí se expone una de ellas, pero estas caricaturas aparecían en cada número, aludiendo siempre al mismo problema. 


\section{IMAGEN 3 \\ Le Rire}

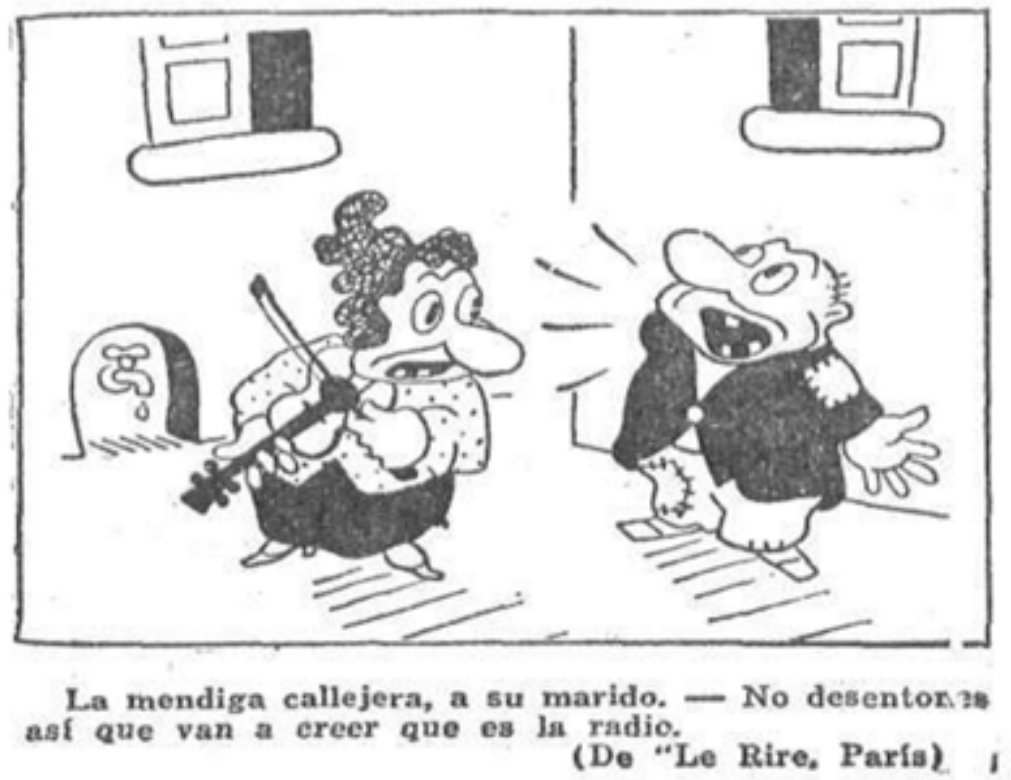

Volviendo a la preocupación de "Radio Cocktail" por la situación generada por Radio Belgrano y la conformación de este "trust de estrellas" alrededor de la emisora de cara a la temporada de 1936, la sección le dedicó durante varios números una serie de notas que dejaban en claro su preocupación por dicho accionar. Pero durante ese año se producirán algunas modificaciones en la sección, que transformarán la interacción de la revista con las emisoras radiales - principalmente con Radio Belgrano-y sus "lectores radioescuchas".

\section{NuEvos diÁLOgOS Y ACERCAMIENTO AL ÉTER}

Los lectores más fieles de Caras y Caretas pueden haber percibido las transformaciones que se dieron en la sección "Radio Cocktail" a mediados de 1936. La temporada radial había comenzado con fuertes críticas por la monopolización de las estrellas que estaba llevando adelante Radio Belgrano. Pero en la edición de fines de febrero de 1936, quienes leían cotidianamente la sección se encontraron con una noticia que se titulaba "Audiciones Caras y Caretas" que decía lo siguiente:

Desde el primero de marzo próximo "Caras y Caretas", por convenio especial con el propietario de Radio Belgrano, señor Jaime Yankelevich, transmitirá todos los lunes una audición especial en horas de la tarde. (...) Radio Belgrano y "Caras y Caretas", unidas en un común propósito de cultura, dirigirán así, sus más nobles y eficientes iniciativas y esfuerzos en un sentido que no podrá menos de ser recibido con el general aplauso y beneplácito del público radioescucha (Caras y Caretas, $\left.1936, \mathrm{~N}^{\circ} 1951\right)$.

La unión entre la prensa escrita y la radio era algo habitual en esa época. El diario La Prensa había adquirido una frecuencia para instalar su emisora, aunque nunca concretó el proyecto. El diario La Nación también tenía su propia emisora y Crítica difundía desde Radio Argentina (Saítta, 2013). Pero lo interesante aquí no es la relación entre ambos medios, sino lo que ello producía en el interior de la propia revista.

Ya mencionamos de qué manera Caras y Caretas se posicionó desde el primer día de la aparición de su sección Radio Cocktail, como defensora de una radiofonía con contenidos culturales y nacionales, y se propuso dirigir sus comentarios hacia ese fin. Lo mismo podemos encontrar en el artículo citado más arriba, titulado "Radio Belgrano y 'Caras y Caretas' unidas en un común propósito de cultura”. Sin embargo, lo relevante no es la aparición de la revista en el espacio del éter, sino las transformaciones que se producen 
dentro de Radio Cocktail, que presentaba artículos y columnas que ya no tenían a Radio Belgrano como la acaparadora de estrellas o cuyos contenidos eran de baja calidad. El eje había cambiado y ahora las miradas no estaban puestas exclusivamente en las emisoras y su funcionamiento. La revista destinó dos hojas por fuera de la sección radial para comentar la programación de sus audiciones en la radio. En la misma, titulada “Transmite ‘Caras y Caretas’ por LR3 Radio Belgrano” exponían:

Este nuevo paso en las actividades de nuestra revista era necesario, ineludible, dado el ritmo moderno que actualmente anima el espíritu de la casa (...) Merced a la gentileza del señor Jaime Yankelevich, director propietario de Radio Belgrano, ha sido posible cristalizar nuestro deseo (Caras y Caretas, 1936, $\mathrm{N}^{\circ}$ 1953. Subrayado de la autora).

Nos encontramos otra vez con una caracterización de la radiofonía inmersa en un nuevo ritmo moderno. La posibilidad de que Caras y Caretas formara parte de ese nuevo espíritu se debía a Yankelevich, quien para esa fecha contaba no solo con el Palacio de las Broadcastings (un edificio que albergaba varias radios a su nombre) sino que también era dueño de la Productora de Cinematografía Argentina Rio de la Plata (Matallana, 2006, 2013). No podemos discernir si se trataba de un proyecto en conjunto para aunar intereses, o si las intenciones eran otras, pero la incorporación de la revista a la programación de dicha radio modificó la forma y los sentidos en los que se hablaba de la radio en la sección Radio Cocktail.

Las discusiones sobre la calidad de los programas de las emisoras o sobre las intenciones de los empresarios de acaparar las mejores estrellas para sus broadcastings, críticas principalmente dirigidas a Radio Belgrano, quedaron en el pasado. La cuestión de la publicidad y de los avisos comerciales cantados tuvo su centralidad después de que la Dirección de Radiocomunicaciones prohibiera su emisión en la radio. Caras y Caretas festejaba: “... desde el $1^{\circ}$ de abril el éter está mucho más limpio. ¡Que te dure, m’hijito!” (Caras y Caretas, $\left.1936, \mathrm{~N}^{\circ} 1958\right)$.

El beneplácito con que la sección comenzó a tratar al dueño de Radio Belgrano y a sus contenidos nos permite retomar el argumento de la historiadora Cecilia Gil Mariño sobre el modelo de convergencia de medios, desarrollado durante esta época. Como se mencionó, Caras y Caretas ocupó a principios de 1930 una posición de acercamiento progresivo y de tono independiente al mundo de la radio. Su origen y su financiación no provenían enteramente de alguna de las emisoras radiales y su contenido estaba dirigido a un amplio campo de la cultura de masas, dentro del cual estaba la sección Radio Cocktail. Pero la relación concretada entre Yankelevich y Caras y Caretas para acceder a un espacio en el éter vino acompañada de una serie de cambios en la mirada sobre la radiofonía en general y sobre Radio Belgrano en particular. Que los nuevos sentidos sobre este medio se matizaran y adquirieran otras valoraciones, no ya del todo críticas, sino explicativas e informativas, nos permite pensar en los condicionantes de la revista para estar en una de las emisoras que más críticas recibía por parte de su sección Radio, al menos en el último tiempo. A partir de la vinculación entre ambas, la columna “ $i N o$ me diga, che...!" solo se ocupaba de informar sobre las nuevas incorporaciones de Radio Belgrano de la siguiente manera:

En Radio Belgrano aseguran la próxima llegada de Raquel Meller para actuar allí con carácter exclusivo. Que en la misma radiodifusora el próximo primero de abril se presentará el prestigioso concertista de violín, León Fontova, frente de una orquesta de 22 profesores (Caras y Caretas, 1936, N 1956).

Al mismo tiempo, la valoración de dicha emisora en el ranking semanal de las broadcastings que señalamos al comienzo se modificó rotundamente en el mes que se anunció la vinculación de Radio Belgrano con la revista. Mientras que el cuadro de Caras y Caretas de febrero de 1936 ubicaba las audiciones de Radio Belgrano en un nivel medianamente bueno (imagen 4), superada solo por Radio Splendid y Radio Fénix, el ranking que aparece en la sección radial del 28/3/1936 se presenta superando el nivel "muy bueno" (imagen 5), y con el resto de las broadcastings porteñas bastante atrás. 
IMAGEN 4

Caras y Caretas de febrero de 1936

NIVEL SEMANAL DE LAS BROADCASTINGS PORTENAS

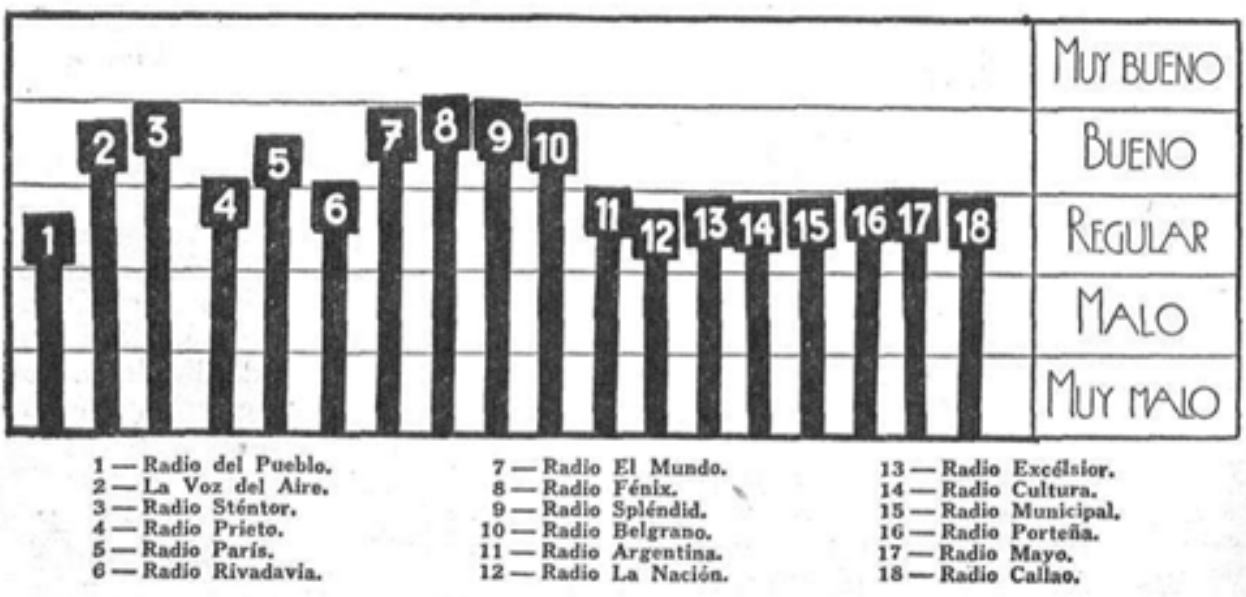

IMAGEN 5

Caras y Caretas de marzo de 1936

NIVEL SEMANAL DE LAS BROADCASTINGS PORTENAS

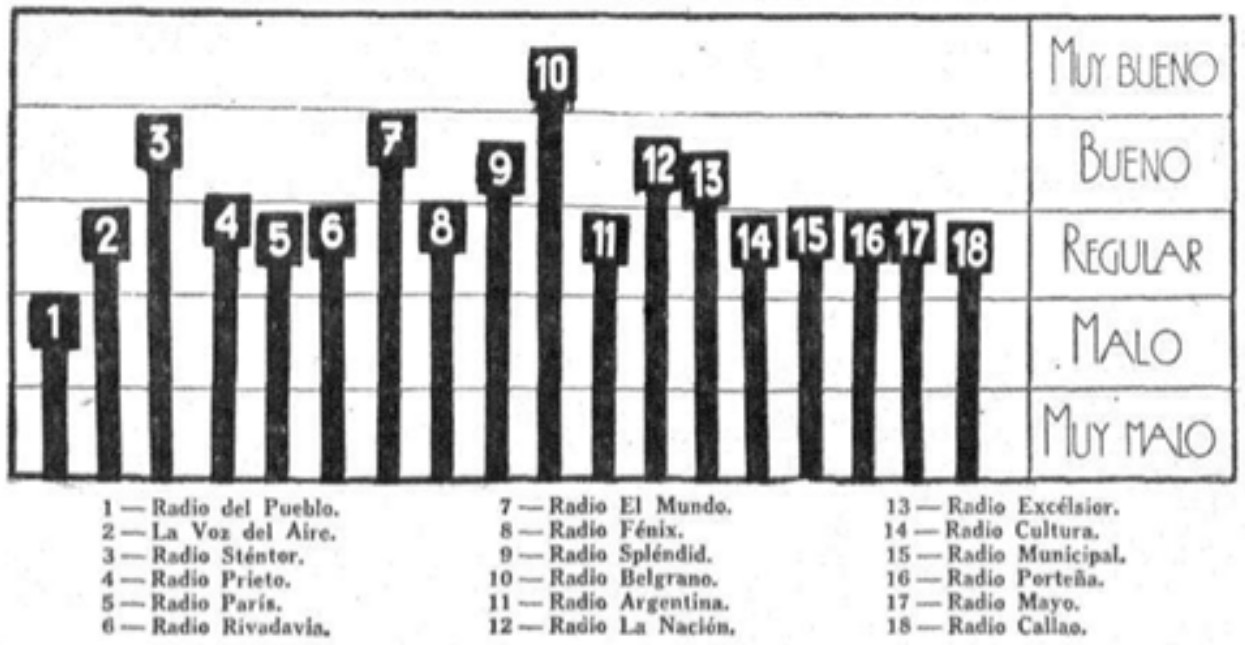

La construcción de este ranking estuvo siempre atravesada por la valoración que la propia Caras y Caretas hacía de los programas que se emitían en las diferentes emisoras. Los intereses a impulsar y promocionar con estas críticas los contenidos que cada una de ellas ponía al aire era una de las tantas maneras de interactuar con sus "lectores radioescuchas". El lugar de intermediaria que ocupaba la sección Radio dentro de la cultura de masas estaba dirigido, como todo medio, a negociar, educar y entablar diálogos entre los intereses de sus lectores y los propios. En otras palabras, el pacto de lectura construido por el magazine buscaba educar a sus lectores en el consumo de programas de "buen gusto" y entretener e informar acerca de las novedades del star system y de las diversas programaciones.

Pero a partir de la asociación con Radio Belgrano vemos que esos intereses propios se relacionan con promover una buena imagen de dicha emisora, o como ellos mismos la caracterizaban: "Radio Belgrano es la broadcasting de los grandes esfuerzos, la empresa que jamás supo retroceder ante ningún obstáculo en su 
permanente afán de servir brillante y honestamente a su público" (Caras y Caretas, 1935, № 1951). De esta forma, los cambios en los intereses de la sección radial se pueden identificar en las diferentes columnas. Solo faltaba un detalle para que las transformaciones de Radio Cocktail se volvieran palpables para los lectores y las lectoras. En julio de 1936 se anunció que Vicente de la Vega era el nuevo director de la sección Radio Cocktail. A partir de allí los cambios fueron contundentes. Para inaugurar el nuevo diseño, la sección comenzó con una presentación titulada "Ayer y hoy" diciendo:

El día 30 de noviembre de 1933, en nuestro afán de reflejar en las páginas de esta revista, "cuanto significara nuevos ritmos de la época, no reñidos con las buenas costumbres ni la cultura del pais", incluímos la "Sección Radio", a los efectos "de dar una de las mejores y más completas informaciones en la materia", pero, luego, el poco interés de los programas y la falta de preocupación de muchos broadcasters, nos obligaron a restarle importancia, ya que nuestras prédicas caían casi siempre en el vacío. Hoy, que la radiodifusión parece haber despertado de su letargo y se vislumbra una promisoria intención de mejoramiento, ampliamos nuevamente la sección... (Caras y Caretas, 1936, N 1972).

Con eso se explicaba que la sección de Radio no había ocupado tantas páginas a mediados de 1934. Los redactores dan cuenta de un fracaso de sus prédicas y su agenda entre las diferentes emisoras porteñas. Pero algo estaba por cambiar a mediados de 1936, que hacía necesario - o que al menos volvía significativo- que la sección Radio Cocktail volviera a ocupar un lugar relevante en Caras y Caretas.

Vicente de la Vega, un hombre conocido del medio que ya había trabajado en teatro, estrenando sus obras en el teatro Maravillas y en la radiofonía, componiendo canciones y obras radioteatrales desde comienzos de la década, le dio una impronta nueva a Radio Cocktail. ${ }^{3}$ El foco de la sección estaba ahora en el star system radial y poco espacio quedaba para mencionar la brutalidad de los speakers o los incumplimientos de las reglamentaciones de la Dirección de Radiocomunicaciones. Las cuestiones de los y las artistas comenzaron a tener mayor importancia y las formas en las se los muestra a través de entrevistas y festejos de camaradería -donde los lectores también participaban - nos habilita a pensar que un nuevo pacto de lectura se había establecido entre la sección y sus lectores.

\section{ConClusión}

A través de este recorrido por la sección Radio hemos dado cuenta de algunas importantes transformaciones que estuvieron atravesadas por dos movimientos: el posicionamiento de una publicación de gran importancia como fue Caras y Caretas en la década de 1930 sobre el nuevo medio, y la consolidación durante ese mismo período de la radio. En los primeros años pudimos observar cómo la sección exponía, en primer lugar, su posición respecto a los valores y contenidos que la radiofonía debía difundir. La visión nacionalista, elitista y defensora de ciertos valores culturales dirigía las críticas más profundas hacia la baja calidad de los programas, que no se correspondían con el "buen gusto" que agradaba a sus lectores.

Pero la sección no solo se encargaba de denunciar las distorsiones de lo que para el magazine era el buen gusto. Las numerosas columnas de chimentos y la búsqueda de satisfacer la curiosidad de sus lectores radioescuchas y entrenarlos como jueces, asumiendo en algunas ediciones la defensa de los intereses del público, respondían a la necesidad de entretener con esas lecturas. Ello se traducía en una convivencia entre la agenda de los redactores y ese público que cada día estaba más interesado en saber cómo funcionaba el medio y quiénes participaban de él. Más allá de las discusiones nacionalistas y estéticas, los redactores debieron construir un pacto de lectura que incluyera los intereses de las lectoras y los lectores para seguir atrayéndolas/ os.

La necesidad de saciar esa curiosidad mediante la descripción de lo que sucedía tras bambalinas de la radio nos permite acceder a las caracterizaciones que los contemporáneos hacían de ese mundo social configurado en torno al medio. A través de las diferentes columnas y artículos analizados pudimos ver cómo, indirectamente, se registraba el funcionamiento del ámbito del trabajo radial, su composición y sus tensiones. 
El interés de los redactores por informar a su público sobre el star system radial, por los conflictos de las emisoras, por la ausencia o presencia de artistas, nos posibilita el acercamiento a ese mundo y la indagación sobre sus configuraciones específicas. Pero al mismo tiempo, esas diversas notas no solo funcionaron como explicaciones sobre lo que los oyentes percibían solo a través de sonidos: también son el registro de la participación de quienes las escribían en las negociaciones - implícitas y no tanto- sobre cómo debía ser representado el fenómeno radial de la década de 1930.

El distanciamiento que la revista impuso en los primeros años de la sección se vio modificado a comienzos de 1936. Un contrato con Jaime Yankelevich le permitió a Caras y Caretas participar del mundo radial, además del editorial. En ese momento, el discurso nacionalista y defensor de la alta cultura, que establecía jerarquías entre los contenidos emitidos por las diferentes emisoras, perdió fuerza y espacio en la sección. Con un nuevo director se inauguró otro discurso, tendiente a exaltar la labor artística del medio y de quienes lo componían, principalmente de Radio Belgrano y de su dueño, el responsable del contrato para que el magazine llegara al público por dicha emisora.

El estudio del caso de Caras y Caretas es interesante para poder analizar sus transformaciones en torno a la relación con la radiofonía. El movimiento desde la crítica, la desconfianza y supuesto desinterés respecto de la firma de un contrato con la propia Radio Belgrano permiten identificar el lugar de importancia que comienza a tener la radio en nuestro país y el interés del magazine por expandir su marca hacia el éter.

\section{ReFERENCIAS DE FUENTES DOCUMENTALES}

Revista Caras y Caretas, Colección Digital, Hemeroteca, Biblioteca Nacional de España:

"Sumario sección Radio", Caras y Caretas, 25/11/33 № 1834.

-"Nuestros propósitos" Caras y Caretas, 2/12/1933 No 1835.

-"La intercalación de avisos", Caras y Caretas, 28 de abril de 1934, № 1856.

-"Entrevista a Carlos Risso Domínguez", Caras y Caretas, 23 de diciembre de 1933, No 1838.

- "Una noche en radio Prieto", Caras y Caretas, 12 de mayo de 1934, № 1858.

“"Audiciones Ernesto Dodds”, Caras y Caretas, 4 de agosto de 1934, № 1870.

-"No me diga, che!", Caras y Caretas, 3 de febrero de 1934, № 1844.

-La uniformidad de los programas", Caras y Caretas, 3 de febrero de 1934, No 1844.

-"Estadísticas”. Caras y caretas, 10 de febrero de 1934, No 1845.

“ ¡No me diga, che!”, Caras y Caretas, 3 de noviembre de 1934, № 1883.

-"Trust de estrellas", Caras y Caretas, 2 de noviembre de 1935, № 1935.

- “?”, Caras y Caretas, 2 de diciembre 1933, № 1835.

- "Las protestas del público", Caras y Caretas, 26 de octubre de 1935, № 1934.

- “ ¡No me dicha, che!”, Caras y Caretas, 5 de octubre de 1935, № 1931.

- “QQué bueno sería!”, Caras y Caretas, 1 de febrero de 1936, No 1948.

- “Audiciones Caras y Caretas”, Caras y Caretas, 22 de febrero de 1936, № 1951.

-“Transmite 'Caras y Caretas' por LR 3 Radio Belgrano”, Caras y Caretas, 7 de marzo de 1936, № 1953.

-"Y vino el sosegate... ", Caras y Caretas, 11 de abril de 1936, No 1958.

- ¡No me diga, che...!”, Caras y Caretas, 28 de marzo de 1936, № 1956.

-"Audiciones Caras y Caretas", Caras y Caretas, 22 de febrero de 1936, № 1951.

-“Ayer y hoy”, Caras y Caretas, 25 de julio de 1936, No 1972.

\section{ReFERENCIAS}

Caimari, L. (2012). Mientras la ciudad duerme. Pistoleros, policias y periodistas en Buenos Aires, 1920-1945. Buenos Aires: Siglo XXI. 
Ehrick, C. (2015). Radio and the gendered soundscape. Women and broadcasting in Argentina and Uruguay, 1930 1950. Nueva York: Cambridge University Press.

Fara, C. (2020). Un horizonte vertical. Paisaje Urbano de Buenos Aires (1910-1936). Buenos Aires: Ampersand.

Gil Mariño, C. (2015). El mercado del deseo. Tango, cine y cultura de masas en la Argentina de los '30. Buenos Aires: Teseo.

Halper, D. (2001). Invisible Stars. A social history of women in American Broadcasting. Nueva York: M. E. Shape.

Liernur, J. F. (2000). La construcción del país urbano. En M. Lobato (ed.), El progreso, la modernización y sus límites (pp. 409-465). Buenos Aires: Sudamericana.

Matallana, A. (2006). Locos por la radio: una historia social de la radiofonía en la Argentina, 1923-1947. Buenos Aires: Prometeo.

Matallana, A. (2013).Jaime Yankelevich: La oportunidady la audacia. Buenos Aires: Capital Intelectual.

Pereira, L. (2016). Negociacões impressas: a imprensa comercial e o lazer dos trabalhadores no Rio de Janeiro da Primeira República. Historia, 35(99), 1-21. http://dx.doi.org/10.1590/1980-436920160000000099

Rogers, G. (2003). Rasgos materiales y mundo de la producción en el semanario Carasy Caretas. Sociohistórica, 13-14, 143-166.

Rogers, G. (2005). Caras y Caretas. Cultura, política y espectáculo en los inicios del siglo XX argentino. Buenos Aires: Editorial de la Universidad de La Plata. Recuperado de: http://sedici.unlp.edu.ar/handle/10915/13724

Rubinzal, M. (2012). El nacionalismo frente a la cuestión social en Argentina [1930-1943]: Discursos, Representaciones $y$ prácticas de las derechas sobre el mundo del trabajo [en línea]. Trabajo final de grado. Universidad Nacional de La Plata. Facultad de Humanidades y Ciencias de la Educación. Recuperado de: http://www.memoria.fahce.u nlp.edu.ar/tesis/te.450/te.450.pdf

Saítta, S. (2013). Regueros de tinta. El diario Crítica en la década de 1920. Buenos Aires: Siglo XXI.

Szir, S. (2009). Entre el arte y la cultura masiva. Las ilustraciones de la ficción literaria en Caras y Caretas (1898-1908). En L. Malosetti Costa y M. Gene (comps.). Impresiones porteñas. Imagen y palabra en la historia cultural de Buenos Aires (pp. 109-139). Buenos Aires: Edhasa.

Yujnovsky, I. (2004). Una vista panorámica de huelgas, manifestaciones y mítines en Caras y Caretas: Prensa y Fotografía a principios del siglo XX en Argentina. América Latina en la Historia Económica, 22, 129-153. http s://doi.org/10.18232/alhe.v11i2.345

\section{Notas}

1 Desde sus comienzos, la sección titulada "Radio Cocktail" publicaba en una columna las distintas críticas de los oyentes, donde era recurrente encontrarse con críticas a los contenidos, a la baja calidad de los programas o a la repetición de las fórmulas por parte de las emisoras.

2 Leonardo Pereira (2016) analizó la polifonía y polisemia presente en los periódicos cariocas de principios de siglo XX.

3 Ver: https://www.todotango.com/creadores/biografia/407/Vicente-de-la-Vega/ 